\title{
Genetic testing for deafness is here, but how do we do it?
}

In this issue of Genetics in Medicine, Schimmenti and colleagues ${ }^{1}$ address several very important issues regarding incorporating genetic testing in the evaluation for newborn hearing impairment (HI). The authors insightfully describe several key points in this process and highlight issues that are both specific to this particular form of genetic testing and have implications for the future. The coming years will see more genetic tests enter the clinical arena for a variety of medical conditions and extend to fields that have not traditionally had a relationship with clinical genetics. The ways in which we address the questions put forth by Schimmenti and colleagues for how genetic testing for newborn $\mathrm{HI}$ is carried out may have implications not only for this one important area but may serve as a model for these future genetic tests. For these reasons, I believe that we as a specialty need to make sure we "get this right" and design a mechanism by which to perform genetic testing for $\mathrm{HI}$ in the most efficient and beneficial manner.

It is important to recognize that we have come to this point in a relatively short time frame. Today, genetic testing for $\mathrm{HI}$ has become an integral component of the evaluation of the deaf and hard of hearing $(\mathrm{D} / \mathrm{HOH})$. However, as recently as 10 years ago, we knew relatively little about the genetic basis of $\mathrm{HI}$. A small number of genes were known for a few rare forms of $\mathrm{HI}$, and clinical testing was not available even for these rare conditions. For most $\mathrm{D} / \mathrm{HOH}$ children and parents, genetics had little to offer. Through family and personal medical history, the goal was to exclude a known hereditary form of HI, or a genetic syndrome that had $\mathrm{HI}$ as one component finding. Although there are several hundred such syndromes, most had other manifestations and could therefore be readily identified. When a diagnosis was established, appropriate management planning and genetic counseling could be offered. However, when the evaluation concluded that the HI was nonsyndromic (NS), recurrence risk counseling relied on empiric data. ${ }^{2}$

Today, genetic testing for $\mathrm{HI}$ is much different. Over 70 HI-related genes have been identified, ${ }^{3}$ but clinical testing is widely available only for deafness at the DFNB1 locus. This type of autosomal recessive deafness is most often caused by mutations in GJB2, the gene that encodes Connexin 26 $(\mathrm{Cx} 26),{ }^{4}$ although a large deletion involving a neighboring gene, GJB6 (encoding Cx30), also causes congenital recessive deafness. ${ }^{6,7}$ Unexpectedly, GJB2 mutations have been found to account for over $55 \%$ of autosomal recessive cases of childhood HI and $30 \%$ of cases with no family history (sporadic cases). ${ }^{5}$ Among North American Caucasians, the carrier frequency for a GJB2 deafness-causing mutation is over 3\% in normal-hearing individuals. ${ }^{5}$ Together, mutations in GJB2 and GJB6 are the most common genetic forms of HI. Mutation screening of these two genes alone will identify an etiology for deafness in up to $50 \%$ of $\mathrm{D} / \mathrm{HOH}$ newborns in developed countries. ${ }^{8}$

This explosion of knowledge about the genetic basis of HI has come at a time of great change in its detection and treatment. Until recently, HI was most commonly identified due to delays in language development, often beyond age 18 months. Even with appropriate therapy, these children suffered irreversible delays in language development, resulting in poor educational outcomes. ${ }^{9}$ It became evident that early detection of HI was the most important factor in a favorable prognosis. Now all states have or will have universal newborn hearing screening, which should greatly increase the likelihood for early detection and intervention of $\mathrm{D} / \mathrm{HOH}$ children. Coupled with improvements in hearing aid and cochlear implant technology, $\mathrm{D} / \mathrm{HOH}$ newborns now have a greater chance of a favorable long-term outcome. The hope and expectation is that genetic testing is one more advance that will benefit these children and their parents. As Schimmenti and colleagues discuss in their article, ${ }^{1}$ however, questions remain regarding the best way to incorporate genetic testing into the early hearing detection and intervention (EHDI) process. They highlight questions concerning the technique of molecular analysis, the best point at which to test, and how follow-up of these tests will be performed. Although complete and thorough, even more practical questions remain.

Few would debate that identifying a genetic cause for $\mathrm{HI}$ provides useful information. As reviewed in the American College of Medical Genetics' Evaluation Guidelines for the Etiologic Diagnosis of Congenital Hearing Loss, ${ }^{10}$ identification of the genetic cause for a patient's HI makes it possible to avoid additional medical tests. Traditional protocols for the evaluation of newborn $\mathrm{HI}$ include a long list of ancillary tests, including thyroid function testing, urinalysis, TORCH titers, and EKG. ${ }^{11}$ Although studies have shown that most of these tests are unnecessary without clinical indication, ${ }^{12}$ most newly diagnosed $\mathrm{D} / \mathrm{HOH}$ patients still have these tests before genetic testing (NHR, personal experience). This illustrates an important and practical problem: it is difficult to get physicians to change the way that they practice, even in the face of established superior protocols.

Other cited benefits of a correct molecular diagnosis are dispelling incorrect notions about the etiology of the HI, accurate recurrence risk counseling, and possibly, limited prognostic information. For example, children with GJB2-related HI typically do well with cochlear implants, and those that have moderate or severe HI tend not to progress. ${ }^{13}$ However, who will convey this information to the parents? Even before that, who 
will do pretest counseling - discuss the test's benefits, limitations, and potential risks (e.g., revealing nonpaternity)?

To begin to answer these questions, we must first ask at what point should genetic testing be introduced into the algorithm of the evaluation of the $\mathrm{D} / \mathrm{HOH}$ newborn? For many of the reasons discussed by Schimmenti et al., ${ }^{1}$ it seems most appropriate to do genetic testing after the $\mathrm{HI}$ is confirmed. Testing at this point would have the best chance to achieve one of the stated objectives of genetic testing for HI, namely, limiting additional unnecessary tests. But testing at this point also creates several potential problems. Who will do the testing and take responsibility for pretest counseling and posttest follow-up, all of which are important if parents are to fully understand the test results? It is probably not reasonable to expect that it will be genetics professionals, simply because patients in many places will not have ready access to them. Will it be the family pediatrician, the otolaryngologist, or the audiologist? All will be intimately involved in the evaluation of these children, but there is reason to doubt that these providers have the training, experience, or time to carry out proper pre- and posttest genetic counseling. However, it will most likely fall to these groups to carry out testing and provide counseling.

Although important, these issues all relate to the health care providers, not the patients. Is this the best point for parents of a newly diagnosed $\mathrm{D} / \mathrm{HOH}$ child to be thinking about the etiology of the HI? Whereas most physicians would think that this is an important question to answer, many audiologists believe that parents are not interested in exploring the genetic aspect at this point (personal communication and experience, 2004). They believe that parents are overwhelmed at the prospect of having a $\mathrm{D} / \mathrm{HOH}$ child, and care more about long-term habilitation and prospects for them to have a normal life. This sentiment echoes what was found in a previous study looking at what the parents of newborns with cleft lip and/or palate (CLP) wanted to know in the newborn period. ${ }^{14}$ It was found that these parents' greatest concerns focused on practical issues such as feeding, and that issues such as etiology and genetics were not as pressing. This question, like many aspects of the process of genetic testing for $\mathrm{HI}$, needs further study before we can be certain if testing at the time of diagnosis is wanted by parents.

Today, clinically available genetic testing for $\mathrm{HI}$ is limited to a few genes, including GJB2, GJB6, SLC26A4, WFS1, and some mitochondrial mutations associated with deafness. However, in the future dozens of HI-related genes will be quickly screened using microarray platforms. Screening for deafness will also include the identification of predisposing alleles for presbycusis (age-related hearing loss) and noise-induced hear- ing loss. Testing of this magnitude will require much more complex counseling. If we are to realize the promise of genomic medicine, for $\mathrm{HI}$ as well as in general, we must get the testing protocols "right" for this first wave, of which genetic testing for $\mathrm{HI}$ is one example. It is therefore imperative to study how to incorporate genetic testing for $\mathrm{HI}$ in a manner that is most beneficial to patients, families, and health care providers. ${ }^{6,7}$

\section{ACKNOWLEDGMENTS}

The author is supported by NIH NIDCD grant 5R03DC006217-02. The author would like to thank Drs. Richard Smith and Monique Ho for their thoughtful comments and suggestions.

Nathaniel H. Robin, MD

The Department of Genetics and Pediatrics University of Alabama at Birmingham Birmingham, Alabama

\section{References}

1. Schimmenti LA, Martinez A, Fox M, Crandall B, Shapiro N, Telatar M et al. Genetic testing as part of the early hearing detection and intervention process. Genet Med 2004;6:521-525.

2. Koehn D, Morgan K, Fraser FC. Recurrence risks for near relatives of children with sensori-neural deafness. Genet Couns 1990;1:127-132.

3. Hereditary hearing loss home page. Available at: http://www.uia.ac.be/dnalab/hhh/ Accessed May 31, 2004

4. Kelsell DP, Dunlop J, Stevens HP, Lench NJ, Liang JN, Parry G et al. Connexin 26 mutations in hereditary non-syndromic sensorineural deafness. Nat Genet 1997; 387:80-83.

5. Green GE, Scott DA, McDonald JM, Woodworth GG, Sheffield VC, Smith RJ. Carrier rates in the midwestern United States for GJB2 mutations causing inherited deafness. JAMA 1999;281:2211-2216.

6. Del Castillo I, Villamar M, Moreno-Pelayo MA, Del Castillo FJ, Alvarez A, Telleria D et al. A deletion involving the connexin 30 gene in non-syndromic hearing impairment. N Engl J Med 2002;346:243-249.

7. Del Castillo I, Moreno-Pelayo MA, Del Castillo FJ, Brownstein Z, Marlin S, Adina Q et al. Prevalence and evolutionary origins of the del(GJB6-D13S1830) mutation in the DFNB1 locus in hearing-impaired subjects: a multicenter study. Am J Hum Genet 2003;73:1452-1458.

8. Prasad S, Cucci RA, Green GE, Smith RJ. Genetic testing for hereditary hearing loss: connexin 26 (GJB2) allele variants and two novel deafness-causing mutations (R32C and 645-648delTAGA). Hum Mut 2000;16:502-508.

9. Yoshaniga-Itano C, Apuzzo ML. Identification of hearing loss after age 18 months is not early enough. Am Ann Deaf; 1998;143:380-387.

10. American College of Medical Geneticists. Genetics evaluation guidelines for the etiologic diagnosis of congenital hearing loss: Genetic evaluation of congenital hearing loss expert panel. ACMG statement. Genet Med 2002;4:162-171.

11. Greinwald JH Jr, Hartnick CJ. The evaluation of children with sensorineural hearing loss. Arch Otolaryngol Head Neck Surg 2002;128:84-87.

12. Parving A, Stephans D. Profound permanent hearing impairment in childhood: causative factors in two European countries. Acta Otol 1997;117:158-160.

13. Green GE, Scott DA, McDonald JM, Teagle HF, Tomblin BJ, Spencer LJ et al. Performance of cochlear implant recipients with GJB2-related deafness. Am J Med Genet 2002;109:167-170.

14. Young JL, O'Riordan M, Goldstein JA, Robin NH. What information do parents of newborns with cleft lip, palate, or both want to know? Cleft Palate Craniofac J 2001; 38:55-58. 